\title{
Nouveaux domaines d'applications et nouvelles techniques
}

\author{
J. Dargent $\cdot$ S. Czernichow \\ (C) Springer-Verlag France 2010
}

Cher(ère) ami(e) lecteur(rice),

Voici notre second numéro « nouvelle formule ». Il comporte deux articles clés sur l'estime de soi et les paramètres anthropométriques en relation avec les recommandations nutritionnelles.

Vous verrez aussi que la chirurgie y occupe davantage de place et que nous avons inséré les abstracts les plus intéressants (ou les plus significatifs des dernières tendances) du Congrès européen de chirurgie de l'obésité (IFSO, Lausanne-Suisse, avril 2010). Nous avons cependant tenu à privilégier ceux qui relevaient le plus de la multidisciplinarité, qui fait actuellement le bonheur de notre spécialité, pour espérons-le, le plus longtemps possible ! S'il était encore besoin d'y insister, mentionnons le succès des dernières Journées médecine et chirurgie de l'obésité, tenues à Paris le 11 juin dernier. Nous ferons de même avec les abstracts du congrès mondial (Los Angeles, septembre), du congrès français (Soffco, Lille, mai 2011) et bien entendu du congrès de l'Association française d'étude et de recherche sur l'obésité
(Afero, Nice, janvier 2011). Également, un article de G. Dapri sur les toutes dernières avancées techniques dans le domaine des opérations bariatriques par « les voies naturelles ». Tous nos lecteurs sont invités à nous envoyer des « cas cliniques médicochirurgicaux» qui feront l'objet de commentaires.

Nous attachons également de l'importance à des revues ou analyses critiques d'articles importants parus récemment : deux publications nord-américaines d'une même équipe ont été ici retenues, ainsi qu'un ouvrage ayant trait à la politique de prévention de l'obésité chez l'enfant.

Enfin, parce qu'aucune discipline n'est à négliger, nous publions un article sur l'art dentaire (parodontaire en l'occurrence) chez l'obèse. Après avoir découvert le rôle majeur du tube digestif comme source de production de nombreuses hormones, celui des interactions entre la gencive, les dents et l'inflammation commence à émerger dans la littérature.

En espérant que vous continuerez à nous soutenir, recevez toute l'expression de notre fidèle amitié !

J. Dargent $(\bowtie) \cdot$ S. Czernichow $(\bowtie)$

e-mail : jerome.dargent@polyclinique-rillieux.fr,

czernichow@uren.smbh.univ-paris13.fr 